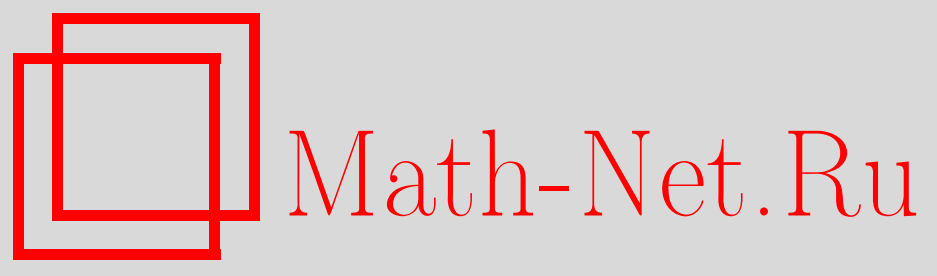

С. С. Марченков, О сложности вычисления рудиментарных предикатов, Дискрет. матем., 2000, том 12, выпуск 4, 83-98

DOI: https://doi.org/10.4213/dm351

Использование Общероссийского математического портала Math-Net.Ru подразумевает, что вы прочитали и согласны с пользовательским соглашением http: //www .mathnet.ru/rus/agreement

Параметры загрузки:

IP : 54.164 .48 .24

26 апреля 2023 г., 18:22:51

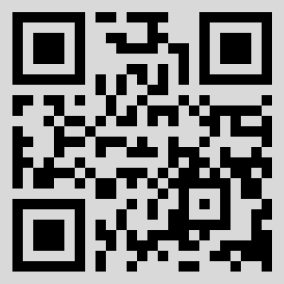




\title{
О сложности вычисления рудиментарных предикатов
}

\author{
(C) 2000 г. $\quad$ C. С. Марченков
}

Класс рудиментарных предикатов определяется как наименьший класс числовых предикатов, который содержит предикаты равенства и конкатенации и замкнут относительно операций логики высказываний, явных преобразований и навешивания ограниченных кванторов. Сложность вычисления рудиментарных предикатов охарактеризовывается в терминах альтернирующих машин Тьюринга с конечным числом альтернирований. Доказывается полиномиальная вычислимость рудиментарных $\exists$ - и $\forall$-предикатов на детерминированных машинах Тьюринга. Определяются иерархии $\left\{R \Sigma_{k}\right\}$ и $\left\{R \Pi_{k}\right\}$ класса рудиментарных предикатов и исследуется класс $R \Sigma_{1} \cap R \Pi_{1}$. Доказывается неразрешимость проблемы непустоты для класса $R \Pi_{1}$.

Работа выполнена при поддержке Российского фонда фундаментальных исследований, проект 00-01-00351.

\section{1. Введение}

Значительное число предикатов, встречающихся в теории алгоритмов, алгебре и теории чисел, можно представить в ограниченно-арифметической форме

$$
\left(Q_{1} y_{1}\right)_{y_{1} \leqslant z_{1}} \ldots\left(Q_{n} y_{n}\right)_{y_{n} \leqslant z_{n}} \rho\left(x_{1}, \ldots, x_{m}, y_{1}, \ldots, y_{n}\right)
$$

где $Q_{1}, \ldots, Q_{n}$ - кванторы $\exists$ или $\forall$, а предикат $\rho$ получается из арифметических предикатов $x+y=z$ и $x \cdot y=z$ с помощью операций логики высказываний и явных преобразований (перестановка и отождествление аргументов, введение фиктивных аргументов, подстановка констант). Класс предикатов, представимых в виде (1), был определен (с небольшими техническими отличиями) А. В. Кузнецовым [1] как класс ограниченно-арифметических предикатов и Р. Смальяном [2] как класс конструктивно-арифметических предикатов. В книге [2] введено также понятие рудиментарного предиката. Совпадение классов ограниченно-арифметических, конструктивно-арифметических и рудиментарных предикатов установлено в [2-5].

Рудиментарные предикаты определяются на множестве натуральных чисел. Предполагается, что натуральные числа взаимно однозначным образом кодируются словами в алфавите $\{1,2\}$ : слову $a_{k} a_{k-1} \ldots a_{1}$, составленному из символов 1 и 2 , сопоставляется натуральное число

$$
\sum_{i=1}^{k} a_{i} 2^{i-1}
$$


Запись $a_{k} a_{k-1} \ldots a_{1}$ называется диадическим представлением числа (2).

В качестве исходных рудиментарных предикатов рассматриваются предикаты равенства $x=y$ и конкатенации $x y=z$ (конкатенация диадических представлений чисел $x$ и $y$ есть диадическое представление числа $z$ ). Класс $\mathbf{R}$ рудиментарных предикатов определяется как наименьший класс предикатов, который содержит предикаты равенства и конкатенации и замкнут относительно операций логики высказываний, явных преобразований и навешивания ограниченных кванторов

$$
(\exists y)_{y \leqslant z}, \quad(\forall y)_{y \leqslant z}
$$

В $[4,5]$ доказано, что классу $\mathbf{R}$ принадлежат предикаты, которые вычислимы на некотором варианте машин Тьюринга за полиномиальное время и с зоной, ограниченной функцией вида $n^{\gamma}$, где $0<\gamma<1$. Ниже, в теоремах 1 и 2 , мы более точно оцениваем сложность вычисления рудиментарных предикатов в терминах альтернирующих машин Тьюринга с конечным числом альтернирований. В теореме 3 положительно решается вопрос о полиномиальном детерминированном вычислении рудиментарных $\exists$ - и $\forall$-предикатов. По аналогии с арифметической иерархией Клини мы определяем иерархии $\left\{R \Sigma_{k}\right\},\left\{R \Pi_{k}\right\}$ класса рудиментарных предикатов и исследуем класс $R \Sigma_{1} \cap R \Pi_{1}$. В теореме 4 устанавливается неразрешимость проблемы непустоты для класса $R \Pi_{1}$. В конце работы в классе $R \Sigma_{2}$ строится пример предиката логарифмического типа.

\section{2. Вычисление рудиментарных предикатов на альтернирующих и детерминированных машинах Тьюринга}

Понятие альтернирующего вычисления введено в [6]. Оно естественным образом обобщает понятие недетерминированного вычисления. Изложим в общих чертах концепцию альтернирующего вычисления применительно к машинам Тьюринга.

Пусть $\mathscr{M}$ - машина Тьюринга произвольного типа с множеством внутренних состояний $Q=\left\{q_{1}, q_{2}, \ldots, q_{s}\right\}$. Будем считать состояние $q_{1}$ начальным состоянием машины $\mathscr{M}$, а $q_{s}-$ ее заключительным состоянием. Функционирование машины $\mathscr{M}$ определяется конечной программой, которая состоит из команд вида

$$
A, q_{i} \rightarrow B, q_{j}
$$

где $A$ - конечный набор символов, которые машина $\mathscr{M}$ может считывать с различных устройств ее внешней памяти (лент, магазинов, счетчиков и т. п.), $B$ конечный набор символов, который задает локальные преобразования внешней памяти. Отметим, что программа машины $\mathscr{M}$ может содержать различные команды с одинаковыми левыми частями.

Предполагается, что множество $Q$ состояний машины $\mathscr{M}$ разбито на два непересекающихся подмножества: множество экзистенциальных состояний и множество универсальных состояний. Обычным образом вводится понятие конфигурации машины $\mathscr{M}$ : конфигурация машины $\mathscr{M}$ состоит из состояния $q_{i}$, а также полного описания состояния внешней памяти машины $\mathscr{M}$ с указанием положения в ней всех считывающе-записывающих устройств. Как правило, в процессе работы машины $\mathscr{M}$ почти вся ее внешняя память является пустой. Поэтому конфигурацию машины 
$\mathscr{M}$ можно задать конечным словом. Конфигурация, содержащая экзистенциальное состояние, называется экзистенциальной, а содержащая универсальное состояние универсальной.

Также обычным образом вводится понятие «конфигурация $K_{2}$ машины $\mathscr{M}$ непосредственно следует за конфигурацией $K_{1}$ » все элементы конфигурации $K_{2}$ должны получаться из соответствующих элементов конфигурации $K_{1}$ за один такт работы машины $\mathscr{M}$. Согласно сделанному выше замечанию о программе машины $\mathscr{M}$, непосредственно за конфигурацией $K_{1}$ может следовать несколько (но конечное число) конфигураций $K_{2}$.

По индукции определим понятие допускающей конфигурации машины $\mathscr{M}$. Каждая конфигурация машины $\mathscr{M}$, содержащая заключительное состояние $q_{s}$ (заключительная конфигурация), считается допускающей конфигурацией машины $\mathscr{M}$. Пусть $K$ - незаключительная конфигурация машины $\mathscr{M}$. Если $K$ - экзистенциальная конфигурация и хотя бы одна из конфигураций машины $\mathscr{M}$, непосредственно следующих за $K$, является допускающей конфигурацией, то $K$ считается допускающей конфигурацией. Двойственным образом, если $K$ - универсальная конфигурация, то конфигурация $K$ считается допускающей, если допускающими являются все конфигурации, которые непосредственно следуют за $K$.

Говорят, что альтернирующая машина $\mathscr{M}$ распознает (вычисляет) предикат $\rho\left(x_{1}, \ldots, x_{n}\right)$, если для любых значений $x_{1}, \ldots, x_{n}$ начальная конфигурация $K\left(x_{1}, \ldots, x_{n}\right)$, отвечающая набору $\left(x_{1}, \ldots, x_{n}\right)$, является допускающей тогда и только тогда, когда истинно значение $\rho\left(x_{1}, \ldots, x_{n}\right)$.

Так же, как для детерминированных машин Тьюринга, для альтернирующих машин Тьюринга можно ввести понятия времени вычисления и зоны вычисления (см. [6]). При этом следует иметь в виду, что в отличие от детерминированного случая альтернирующие вычисления можно представить в виде ориентированного дерева, в корне которого находится начальная конфигурация, а в конечных вершинах - заключительные конфигурации. Поэтому время альтернирующего вычисления представляет собой длину наибольшей цепи в этом дереве, а зона альтернирующего вычисления равна максимуму из зон конфигураций, составляющих дерево.

Пусть $k \geqslant 0$. Дерево вычисления на альтернирующей машине $\mathscr{M}$ называется $k$ альтернирующим, если каждая цепь дерева вычисления содержит не более $k$ перемен экзистенциальных и универсальных конфигураций. Соответствующим образом, машина $\mathscr{M}$ называется $k$-альтернирующей, если каждое вычисление на машине $\mathscr{M}$ является $k$-альтернирующим.

Ниже, в теоремах 1 и 2, будет рассматриваться следующий вариант машин Тьюринга (см. также $[4,5])$. Машина Тьюринга имеет две ленты: входную и рабочую. На входной ленте помещается читающая головка, которая может двигаться по ленте в обе стороны, не выходя за пределы записанного на ленте слова и не меняя записанных на ленте символов. На рабочей ленте располагается головка, которая может двигаться в обе стороны, читать записанные и записывать новые символы. Алфавит входной ленты состоит из символов $1,2, *$. При вычислении на машине предиката $\rho\left(x_{1}, \ldots, x_{n}\right)$ от аргументов $x_{1}, \ldots, x_{n}$, принимающих натуральные значения, набор $\left(x_{1}, \ldots, x_{n}\right)$ представляется на входной ленте в виде

$$
* d\left(x_{1}\right) * d\left(x_{2}\right) * \ldots * d\left(x_{n}\right) *
$$

где $d\left(x_{i}\right)$ - диадическое представление $x_{i}$. В начальный момент вычисления головка на входной ленте устанавливается на самый левый символ $*$. 
Алфавит рабочей ленты состоит из символов $b_{0}, b_{1}, \ldots, b_{r}$, где $b_{0}-$ пустой символ. В начальный момент вычисления рабочая лента пуста (состоит из одних символов $\left.b_{0}\right)$.

Программа машины состоит из команд вида

$$
a b_{j} q_{k} \rightarrow b_{m} M N q_{l}
$$

где $a \in\{1,2, *\}, k \neq s, M, N \in\{L, R, S\}$. Команда (3) интерпретируется следующим образом. Если в момент $t$ машина находится в состоянии $q_{k}$, головки на входной и рабочей лентах обозревают соответственно символы $a$ и $b_{j}$, то в момент $t+1$ машина будет находиться в состоянии $q_{l}$, символ $b_{j}$ на рабочей ленте будет заменен символом $b_{m}$, а головки на входной и рабочей лентах переместятся в соответствии с символами $M$ и $N$ ( $L$ - сдвиг на одну позицию влево, $R$ - вправо, $S$ - отсутствие сдвига).

Пусть в момент времени $t$ машина Тьюринга $\mathscr{M}$ указанного типа находится в состоянии $q_{i}$, на ее входной ленте записано слово $u$, головка на входной ленте обозревает $j$-ю букву слова $u, v$ - слово, записанное на рабочей ленте слева от головки (крайние слева символы $b_{0}$ в слове $v$ опускаются, исключение составляет случай $\left.v=b_{0}\right), w$ - аналогичное слово, расположенное справа от головки, причем в момент $t$ машина $\mathscr{M}$ обозревает первую букву слова $w$. Тогда набор $(i, u, j, v, w)$ называется конфигурацией машины $\mathscr{M}$ в момент $t$. Зона вычисления на машине $\mathscr{M}$ определяется по рабочей ленте.

Теорема 1. Пусть рудиментарный предикат $\sigma\left(x_{1}, \ldots, x_{m}, z_{1}, \ldots, z_{n}\right)$ представим в форме (1), где кванторная приставка $Q_{1} \ldots Q_{n}$ содержит $k$ перемен кванторов, а предикат $\rho$ построен из предикатов равенства и конкатенации с помощъю операций логики высказываний и явных преобразований.

Тогда предикат $\sigma$ можно въчислитъ на подходящей $k$-алътернирующей машине Тъюринга за не более чем квадратичное время и слиейной зоной.

Доказательство. Опишем неформальным образом работу $k$-альтернирующей машины Тьюринга $\mathscr{M}$, которая вычисляет предикат $\sigma$. Эта работа естественно разбивается на два этапа. На первом этапе с помощью экзистенциальных и универсальных состояний происходит выбор надлежащих значений аргументов $y_{1}, \ldots, y_{n}$ в формуле (1). На втором этапе для заданных значений аргументов $x_{1}, \ldots, x_{m}$ и найденных значений аргументов $y_{1}, \ldots, y_{n}$ проводится вычисление значения $\rho\left(x_{1}, \ldots, x_{m}, y_{1}, \ldots, y_{n}\right)$.

Более подробно, пусть для определенности в кванторной приставке $Q_{1} \ldots Q_{n}$ первые $p$ кванторов суть кванторы существования, следующие $q$ кванторов суть кванторы общности и т. д. Используя экзистенциальные состояния, машина $\mathscr{M}$ записывает на рабочей ленте какие-либо значения $y_{1}, \ldots, y_{p}$, подчиняющиеся условиям $y_{1} \leqslant z_{1}, \ldots, y_{n} \leqslant z_{n}$. Процесс записи набора $\left(y_{1}, \ldots, y_{p}\right)$ на рабочей ленте требует не более чем квадратичного (относительно длины записи набора $\left(z_{1}, \ldots, z_{p}\right)$ ) времени и линейной зоны, поскольку разряды чисел $y_{1}, \ldots, y_{p}$ просто последовательно угадываются. Угадав некоторый набор $\left(y_{1}, \ldots, y_{p}\right)$, машина $\mathscr{M}$ переходит в блок универсальных состояний и аналогичным образом за не более чем квадратичное время и на линейной зоне генерирует наборы $\left(y_{p+1}, \ldots, y_{p+q}\right)$. Затем машина $\mathscr{M}$ переходит в блок экзистенциальных состояний и т. д. Таким образом, весь процесс отыскания значений аргументов $y_{1}, \ldots, y_{n}$ занимает не более чем квадратичное время и выполняется на линейной зоне. 
Второй этап работы машины $\mathscr{M}$ полностью детерминирован. Здесь происходит вычисление предикатов вида

$$
v_{1}=v_{2}, \quad \neg\left(v_{1}=v_{2}\right), \quad v_{1} v_{2}=v_{3}, \quad \neg\left(v_{1} v_{2}=v_{3}\right),
$$

где $v_{1}, v_{2}, v_{3}$ - значения аргументов $x_{1}, \ldots, x_{m}, y_{1}, \ldots, y_{n}$, либо константы, входящие в предикат $\rho$. Очевидно, что проверку выполнения равенств $v_{1}=v_{2}$ и $v_{1} v_{2}=v_{3}$ можно выполнить за не более чем квадратичное время и на линейной зоне. Теорема доказана.

Теорема 2. Пусть $k \geqslant 1,0<\gamma<1$ и предикат $\rho$ вычислим $k$-альтернирующей машиной Тъюринга за полиномиальное времл $и$ на зоне, ограниченной бунхцией вида $n^{\gamma}$. Тогда $\rho$ естъ рудиментарный предикат.

Доказательство теоремы 2 почти дословно повторяет доказательство теоремы 1 из [4] (детерминированный вариант теоремы 2) и теоремы 1 из [5] (недетерминированный вариант теоремы 2). Поэтому мы его опускаем.

Назовем рудиментарный предикат $\exists$-предикатом, если его можно представить в форме (1), где

$$
Q_{1}=\ldots=Q_{n}=\exists
$$

и предикат $\rho$ построен из предикатов равенства и конкатенации с помощью операций логики высказываний и явных преобразований. Аналогичным образом определяем рудиментарный $\forall$-предикат. Легко видеть, что отрицанием рудиментарного $\exists$-предиката является рудиментарный $\forall$-предикат и наоборот.

Если рудиментарные предикаты вычислять непосредственно по формуле (1), то, вообще говоря, время вычисления будет зависеть экспоненциальным образом от длины записи аргументов. Теорема 1 из [5] и теорема 2 настоящей работы оставляют мало надежд на то, что эту тривиальную экспоненциальную оценку удастся понизить до полиномиальной. Поэтому на данном этапе исследований класса $\mathbf{R}$ представляет интерес выделение в $\mathbf{R}$ как можно более широких классов предикатов, для которых имеется полиномиальная верхняя оценка времени детерминированного вычисления. Теорема 3 , доказываемая ниже, говорит о том, что к таким классам относятся классы рудиментарных $\exists$ - и $\forall$-предикатов.

Говоря здесь и далее о полиномиальном времени вычисления и о полиномиальных алгоритмах, мы имеем в виду, что выбран достаточно широкий класс абстрактных вычислительных устройств (например, этими устройствами могут быть многоленточные машины Тьюринга [7]) и полиномиальные алгоритмы реализуются детерминированно за полиномиальное (от длины входа) время в этом классе вычислительных устройств. Что касается вопроса о представлении в этих устройствах рассматриваемых ниже формул, то, не углубляясь в технические детали, будем предполагать, что выбран некоторый естественный способ кодирования формул словами некоторого конечного алфавита.

Прежде чем переходить к теореме 3 , установим одно вспомогательное утверждение технического характера. Как обычно, предикаты $\neg(x=y)$ и $\neg(x y=z)$ будем обозначать через $x \neq y$ и $x y \neq z$.

Лемма 1. Предикаты $x \neq y$ u $x y \neq z$ можно получить из предикатов равенства и конкатенации с помощъю логических операчий конбюнкции и дизвюнкции, явных преобразований и навешивания ограниченных кванторов существования. 
Доказательство. Мы рассмотрим только предикат $x y \neq z$. Предикат $x \neq y$ получается аналогично более простыми построениями.

Очевидно, что слово $x y$ отлично от слова $z$ в том и только том случае, когда выполняется хотя бы одно из условий.

(1) Слово $x y$ есть собственное начало слова $z$.

(2) Слово $z$ есть собственное начало слова $x y$.

(3) Существует такое слово $v$, которое является собственным началом слов $x y$ и $z$, при этом для некоторого символа $a, a \in\{1,2\}$, слово $v a$ есть начало (не обязательно собственное) слова $x y$, а слово $v b$, где $b \in\{1,2\}$ и $b \neq a,-$ начало (тоже не обязательно собственное) слова $z$.

Условие 2 естественным образом разбивается на три следующих попарно не пересекающихся подслучая:

(2а) Слово $z$ есть собственное начало слова $x$.

(2b) $z=x$.

(2c) Слово $z$ есть конкатенация слова $x$ и собственного начала слова $y$.

В свою очередь условие 3 также можно разбить на три попарно не пересекающихся подслучая:

(3a) Слово $v$ есть собственное начало слова $x$.

(3b) $v=x$.

(3c) Слово $v$ есть конкатенация слова $x$ и собственного начала слова $y$.

Каждому из пп. 1, 2a-2c, 3a-3c будет сопоставлена формула рассматриваемого в лемме типа, которая определяет описанный в условии пункта предикат. Дизъюнкция этих семи формул и будет задавать предикат $x y \neq z$.

(1) $\left(\exists v_{1}\right)_{v_{1} \leqslant z}\left(\exists v_{2}\right)_{v_{2} \leqslant z}\left(\left(x y=v_{1}\right) \&\left(v_{1} v_{2}=z\right)\right)$.

(2a) $(\exists v)_{v \leqslant x}(z v=x)$.

(2b) $z=x$.

(2c) $\left(\exists v_{1}\right)_{v_{1} \leqslant y}\left(\exists v_{2}\right)_{v_{2} \leqslant y}\left(\left(x v_{1}=z\right) \&\left(v_{1} v_{2}=y\right)\right)$.

В случае 3а формула имеет вид

$$
\begin{gathered}
(\exists v)_{v \leqslant x}\left(\exists v_{1}\right)_{v_{1} \leqslant x}\left(\exists v_{2}\right)_{v_{2} \leqslant z} \\
\left(\left(v 1=x \vee v 1 v_{1}=x\right) \&\left(v 2=z \vee v 2 v_{2}=z\right) \vee\left(v 2=x \vee v 2 v_{1}=x\right) \&\left(v 1=z \vee v 1 v_{2}=z\right)\right)
\end{gathered}
$$

(встречающиеся в этой формуле предикаты типа $v 1 v_{1}=x$ необходимо представить в форме

$$
\left.\left(\exists v_{3}\right)_{v_{3} \leqslant x}\left(\left(v 1=v_{3}\right) \&\left(v_{3} v_{1}=x\right)\right)\right)
$$


В случае $3 \mathrm{~b}$ формула имеет вид

$$
\begin{aligned}
& \left(\exists v_{1}\right)_{v_{1} \leqslant y}\left(\exists v_{2}\right)_{v_{2} \leqslant z} \\
& \left(\left(1=y \vee 1 v_{1}=y\right) \&\left(x 2=z \vee x 2 v_{2}=z\right) \vee\left(2=y \vee 2 v_{1}=y\right) \&\left(x 1=z \vee x 1 v_{2}=z\right)\right)
\end{aligned}
$$

Наконец, в случае 3с формула имеет вид

$$
\begin{array}{r}
\left(\exists v_{1}\right)_{v_{1} \leqslant y}\left(\exists v_{2}\right)_{v_{2} \leqslant y}\left(\exists v_{3}\right)_{v_{3} \leqslant z}\left(\left(v_{1} 1=y \vee v_{1} 1 v_{2}=y\right) \&\left(x v_{1} 2=z \vee x v_{1} 2 v_{3}=z\right)\right. \\
\left.\vee\left(v_{1} 2=y \vee v_{1} 2 v_{2}=y\right) \&\left(x v_{1} 1=z \vee x v_{1} 1 v_{3}=z\right)\right)
\end{array}
$$

Лемма доказана.

Теорема 3. Любой рудиментарный Э-предикат можно вычислить подходящим детерминированным полиномиальным алгоритмом.

Доказательство. Пусть рудиментарный Э-предикат

$$
\sigma\left(x_{1}, \ldots, x_{m}, z_{1}, \ldots, z_{n}\right)
$$

представим в форме (1), где $Q_{1}=\ldots=Q_{n}=\exists$ и предикат $\rho$ получается из предикатов равенства и конкатенации с помощью операций логики высказываний и явных преобразований. Будем предполагать, что в качестве операций логики высказываний выбраны операции $\&, \vee, \neg$. Согласно доказанной лемме операцию $\neg$ из этого перечня можно исключить. После приведения предиката $\rho$ к дизъюнктивной нормальной форме получим, что

$$
\sigma\left(x_{1}, \ldots, x_{m}, z_{1}, \ldots, z_{n}\right) \equiv\left(\exists y_{1}\right)_{y_{1} \leqslant z_{1}} \ldots\left(\exists y_{n}\right)_{y_{n} \leqslant z_{n}}\left(K_{1} \vee \ldots \vee K_{p}\right)
$$

где $K_{1}, \ldots, K_{p}$ - конъюнкции предикатов типа

$$
u=v, \quad u v=w, \quad u=a, \quad u a=w, \quad a u=w, \quad u v=a, \quad u a=b, \quad a u=b,
$$

$u, v, w$ - переменные из множества $\left\{x_{1}, \ldots, x_{m}, y_{1}, \ldots, y_{n}\right\}, a, b$ - константы. Согласно известным логическим правилам, правая часть формулы (4) эквивалентна формуле

$$
\left(\exists y_{1}\right)_{y_{1} \leqslant z_{1}} \ldots\left(\exists y_{n}\right)_{y_{n} \leqslant z_{n}} K_{1} \vee \ldots \vee\left(\exists y_{1}\right)_{y_{1} \leqslant z_{1}} \ldots\left(\exists y_{n}\right)_{y_{n} \leqslant z_{n}} K_{p}
$$

Следовательно, при оценке сложности вычисления предиката $\sigma$ можно ограничиться рассмотрением лишь предикатов вида

$$
\left(\exists y_{1}\right)_{y_{1} \leqslant z_{1}} \ldots\left(\exists y_{n}\right)_{y_{n} \leqslant z_{n}} K
$$

где конъюнкция $K$ подчиняется сформулированным выше условиям для конъюнкций $K_{1}, \ldots, K_{p}$.

Пусть $l_{1}=\left|z_{1}\right|, \ldots, l_{n}=\left|z_{n}\right|$, где $\left|z_{i}\right|$ - длина слова $z_{i}$. Область изменения каждой переменной $y_{i}$ в формуле (6) разобьем на $l_{i}$ попарно не пересекающихся областей

$$
\left|y_{i}\right|=1, \ldots,\left|y_{i}\right|=l_{i}-1,\left(\left|y_{i}\right|=l_{i}, y_{i} \leqslant z_{i}\right)
$$


Соответственно этому разбиению каждый квантор $\left(\exists y_{i}\right)_{y_{i} \leqslant z_{i}}$ в формуле (6) заменим на $l_{i}$ ограниченных кванторов

$$
\left(\exists y_{i}\right)_{\left|y_{i}\right|=1}, \ldots,\left(\exists y_{i}\right)_{\left|y_{i}\right|=l_{i}-1},\left(\exists y_{i}\right)_{\left|y_{i}\right|=l_{i}, y_{i} \leqslant z_{i}} .
$$

В результате формулу (6) можно эквивалентным образом представить в виде дизъюнкции $l_{1} \ldots l_{n}$ формул, каждая из которых имеет вид

$$
\left(\exists y_{1}\right)_{y_{1} \in D_{1}} \ldots\left(\exists y_{n}\right)_{y_{n} \in D_{n}} K
$$

где для любого $i, 1 \leqslant i \leqslant n$, множество $D_{i}$ определяется одним из условий (7). Величина $l_{1} \ldots l_{n}$, очевидно, ограничена сверху полиномом степени $n$ от $l_{1}+\ldots+l_{n}$, то есть от длины записи аргументов $z_{1}, \ldots, z_{n}$. Поэтому в дальнейшем будем оценивать лишь сложность вычисления предикатов (8).

Рассмотрим множество $D_{i}$, когда $\left|y_{i}\right|=l_{i}, y_{i} \leqslant z_{i}$ и $z_{i} \neq 22 \ldots 2$. Пусть $y_{1}^{i}, \ldots, y_{l_{i}}^{i}$ - разряды диадического представления $y_{i}, z_{1}^{i}, \ldots, z_{l_{i}}^{i}$ - аналогичные разряды представления $z_{i}$. Если величина $z_{i}$ фиксирована, $r_{1}, \ldots, r_{s}$ - номера всех разрядов слова $z_{l_{i}}^{i} \ldots z_{1}^{i}$, равных 2 , и $r_{1}<\ldots<r_{s}$, то множество $D_{i}$ разбиваем на $s+1$ попарно не пересекающихся подмножеств:

$$
\begin{aligned}
D_{i}^{1} & =\left\{y_{i}: y_{i} \in D_{i}, y_{r_{1}}^{i}=1, y_{r_{1}+1}^{i}=z_{r_{1}+1}^{i}, \ldots, y_{l_{i}}^{i}=z_{l_{i}}^{i}\right\} \\
& \ldots \\
D_{i}^{s} & =\left\{y_{i}: y_{i} \in D_{i}, y_{r_{s}}^{i}=1, y_{r_{s}+1}^{i}=z_{r_{s}+1}^{i}, \ldots, y_{l_{i}}^{i}=z_{l_{i}}^{i}\right\}, \\
D_{i}^{s+1} & =\left\{z_{i}\right\} .
\end{aligned}
$$

Отметим одну особенность строения множеств $D_{i}^{1}, \ldots, D_{i}^{s}$, которая легко усматривается из определения. Двоичные разряды $y_{1}^{i}, \ldots, y_{l_{i}}^{i}$ чисел $y_{i}$, составляющих множество $D_{i}^{t}, 1 \leqslant t \leqslant s$, делятся на две группы: в первой группе (разряды $y_{r_{t}}^{i}, y_{r_{t}+1}^{i} \ldots, y_{l_{i}}^{i}$ ) они фиксированы (и, за исключением разряда $y_{r_{t}}^{i}$, совпадают с соответствующими разрядами числа $z_{i}$ ), во второй группе (разряды $y_{1}^{i}, \ldots, y_{r_{t}-1}^{i}$ ) произвольны. Множество $D_{i}^{s+1}$ состоит из одного числа $z_{i}$, поэтому при $y_{i} \in D_{i}^{s+1}$ все двоичные разряды числа $y_{i}$ фиксированы.

Если величина $z_{i}$ фиксирована, а в формулу (8) входит квантор $\left(\exists y_{i}\right)_{y_{i} \in D_{i}}$, где множество $D_{i}$ определяется последней из формул (7), то этот квантор можно заменить последовательностью из $s+1$ ограниченных кванторов

$$
\left(\exists y_{i}\right)_{y_{i} \in D_{i}^{1}}, \ldots,\left(\exists y_{i}\right)_{y_{i} \in D_{i}^{s+1}}
$$

Таким образом, при фиксированных значениях аргументов $z_{1}, \ldots, z_{n}$ формула (8) эквивалентна дизъюнкции не более $l_{1} \ldots l_{n}$ формул вида

$$
\left(\exists y_{1}\right)_{y_{1} \in E_{1}} \ldots\left(\exists y_{n}\right)_{y_{n} \in E_{n}} K
$$

где для любого $i, 1 \leqslant i \leqslant n$, множество $E_{i}$ совпадает либо с одним из первых $l_{i}-1$ множеств ряда (7), либо с одним из множеств (9). Поэтому при вычислении предиката $\sigma$ можно ограничиться рассмотрением лишь формул вида (10).

Пусть $x_{k_{i}}^{i} \ldots x_{1}^{i}$ - диадическое представление $x_{i}, 1 \leqslant i \leqslant m$. Предположим, что значения всех аргументов $x_{1}, \ldots, x_{m}, z_{1}, \ldots, z_{n}$ фиксированы,

$$
x_{1}=a_{1}, \ldots, x_{m}=a_{m}, \quad z_{1}=b_{1}, \ldots, z_{n}=b_{n} .
$$


Покажем, как проверить истинность предиката (10).

Заменим в конъюнкции $K$ каждый из входящих в нее предикатов (5) его аналогом, полученным из указанных представлений аргументов $y_{1}, \ldots, y_{n}$ и констант $a_{1}, \ldots, a_{m}, b_{1}, \ldots, b_{n}$. Например, предикаты $x_{1} y_{1}=y_{2}$ и $x_{1} x_{2}=y_{1}$ следует заменить соответственно предикатами

$$
a_{k_{1}}^{1} \ldots a_{1}^{1} y_{k}^{1} \ldots y_{1}^{1}=y_{l}^{2} \ldots y_{1}^{2}, \quad a_{k_{1}}^{1} \ldots a_{1}^{1} a_{k_{2}}^{2} \ldots a_{1}^{2}=y_{k}^{1} \ldots y_{1}^{1}
$$

где $k, l$ - размерности множеств $E_{1}, E_{2}$. Заметим, что $y_{k}^{1}, \ldots, y_{1}^{1}$ и $y_{l}^{2}, \ldots, y_{1}^{2}$ могут быть как двоичными переменными, так и двоичными константами, определяемыми константами $b_{1}$ и $b_{2}$. Если, например, $k<l_{1}$, то $y_{k}^{1}, \ldots, y_{1}^{1}$ суть двоичные переменные. В противном случае для некоторого $r, r<l_{1}, y_{r-1}^{1}, \ldots, y_{1}^{1}$ являются двоичными переменными, $y_{r}^{1}=1$ и $y_{l_{1}}^{1}, \ldots, y_{r+1}^{1}$ совпадают соответственно с $b_{l_{1}}^{1}, \ldots, b_{r+1}^{1}$.

В результате этой замены могут образоваться противоречивые равенства. Например, в формулах (11) могут либо не выполняться равенства $k_{1}+k=l$ или $k_{1}+k_{2}=k$, либо некоторая двоичная константа из левой части одного из равенств (11) может не совпадать с стоящей на соответствующей позиции двоичной константой из правой части равенства. В этом случае, разумеется, формула (10) при заданных значениях $x_{1}, \ldots, x_{m}, z_{1}, \ldots, z_{n}$ будет ложной.

Предполагая, что равенства типа (11) не содержат противоречий, заменим каждое из них эквивалентной системой равенств вида

$$
y_{j}^{i}=y_{q}^{t}, \quad y_{j}^{i}=c
$$

где $c$ - двоичная константа. Образуем транзитивное замыкание $W$ совокупности всех равенств (12), построенных для всех подформул (5) конъюнкции $K$. Это множество $W$ может также оказаться противоречивым, содержа одновременно равенства вида $y_{j}^{i}=c, y_{j}^{i}=d$, где $c \neq d$. Тогда будет ложным и предикат (10). В противном случае множество $W$ разбивается на непротиворечивые классы эквивалентности, каждый из которых имеет вид

$$
v_{1}=\ldots=v_{q} \quad \text { или } \quad v_{1}=\ldots=v_{q}=c
$$

где $v_{1}, \ldots, v_{q}$ - двоичные $y$-переменные, $c$ - двоичная константа (истинные равенства вида $c=c$ опускаем). В этом случае истинность предиката (10) эквивалентна существованию значений двоичных $y$-переменных, удовлетворяющих равенствам (13). Поскольку каждая из формул

$$
\left(\exists v_{1}\right) \ldots\left(\exists v_{q}\right)\left(v_{1}=\ldots=v_{q}\right), \quad\left(\exists v_{1}\right) \ldots\left(\exists v_{q}\right)\left(v_{1}=\ldots=v_{q}=c\right)
$$

истинна, истинным будет и значение предиката (10). Теорема доказана.

Следствие 1. Любой рудиментарный $\forall$-предикат можно вычислить подходящим детерминированным полиномиальным алгоритмом.

Достаточно заметить, что отрицание рудиментарного $\forall$-предиката является рудиментарным $\exists$-предикатом. 


\section{3. Иерархия рудиментарных предикатов}

Как установлено в [5], при определении рудиментарных предикатов соотношения вида $y \leqslant z$, фигурирующие в ограниченных кванторах, можно заменить более общими соотношениями вида $y \leqslant T$, где $T$ - произвольный словарный терм, составленный из слов в алфавите $\{1,2\}$ и символов переменных. В связи с этим элементарными рудиментарными предикатами естественно считать все предикаты вида $T=V$, где $T, V$ - произвольные словарные термы. Класс всех рудиментарных предикатов, полученных из этих элементарных предикатов с помощью операций логики высказываний, обозначим через $R \Sigma_{0}$. Положим $R \Pi_{0}=R \Sigma_{0}$. Для любого $k, k \geqslant 0$, определим $R \Sigma_{k+1}$ (соответственно $R \Pi_{k+1}$ ) как класс всех рудиментарных предикатов, представимых в форме

$$
\left(Q y_{1}\right)_{y_{1} \leqslant T_{1}} \ldots\left(Q y_{n}\right)_{y_{n} \leqslant T_{n}} \rho\left(x_{1}, \ldots, x_{m}, y_{1}, \ldots, y_{n}\right)
$$

где $Q=\exists$ (соответственно $Q=\forall), T_{1}, \ldots, T_{n}$ - словарные термы и $\rho$ - предикат из класса $R \Pi_{k}$ (соответственно $\left.R \Sigma_{k}\right)$. Положим

$$
R \Delta_{k}=R \Sigma_{k} \cap R \Pi_{k}
$$

Как и для арифметической иерархии Клини (см. также словарную иерархию в [8]), имеют место соотношения

$$
\begin{aligned}
& R \Sigma_{0} \subseteq R \Sigma_{1} \subseteq R \Sigma_{2} \subseteq \ldots, \quad R \Pi_{0} \subseteq R \Pi_{1} \subseteq R \Pi_{2} \subseteq \ldots, \\
& R \Sigma_{k} \cup R \Pi_{k} \subseteq R \Delta_{k+1}, \quad \rho \in R \Sigma_{k} \Longleftrightarrow \neg \rho \in R \Pi_{k}, \quad k=0,1, \ldots
\end{aligned}
$$

Если предикаты $\rho, \sigma$ принадлежат классу $R \Sigma_{k}$ (соответственно $R \Pi_{k}$ ), то этому же классу принадлежат предикаты $\rho \& \sigma$ и $\rho \vee \sigma$.

Используя технику работы [5] или технику доказательства леммы из предыдущего раздела, можно показать, что класс рудиментарных $\exists$-предикатов совпадает с классом $R \Sigma_{1}$, а класс рудиментарных $\forall$-предикатов - с классом $R \Pi_{1}$.

Если $a \in\{1,2\}$, то пусть $\alpha_{a}(x)$ обозначает предикат «слово $x$ состоит только из символов $a »$. Очевидно, что

$$
\alpha_{a}(x) \equiv(a x=x a)
$$

Поэтому $\alpha_{a} \in R \Sigma_{0}$ и $\alpha_{a} \in R \Pi_{0}$.

Определим ряд предикатов, каждый из которых принадлежит классу $R \Delta_{1}$. Cправедливы соотношения

$$
(x \leqslant y) \equiv(\exists z)_{z \leqslant y}(x=z), \quad(x<y) \equiv(x \leqslant y) \&(x \neq y) .
$$

Следовательно, предикаты $x \leqslant y, x<y$ входят в класс $R \Sigma_{1}$. Формулы

$$
(x<y) \equiv(\forall z)_{z \leqslant x}(y \neq z), \quad(x \leqslant y) \equiv(x<y) \vee(x=y)
$$

показывают, что предикаты $x \leqslant y, x<y$ входят также в класс $R \Pi_{1}$.

Пусть $|x|$ есть длина диадического представления $x$. Следующие две эквивалентности устанавливают принадлежность предиката $|x|=|y|$ классу $R \Delta_{1}$ :

$$
\begin{aligned}
& (|x|=|y|) \equiv(\exists z)_{z \leqslant x}\left(\alpha_{1}(z) \&(z \leqslant y) \&(x<1 z) \&(y<1 z)\right), \\
& (|x|=|y|) \equiv(\forall u)_{u \leqslant x}(\forall v)_{v \leqslant y}\left(\left(\alpha_{1}(u) \& \alpha_{1}(v) \&(x<1 u) \&(y<1 v) \Rightarrow(u=v)\right) .\right.
\end{aligned}
$$


На базе предиката $|x|=|y|$ доказываем вхождение предикатов $|x|<|y|$ и $|x| \leqslant|y|$ сначала в класс $R \Sigma_{1}$,

$$
(|x| \leqslant|y|) \equiv(\exists z)_{z \leqslant y}(|x|=|z|), \quad(|x|<|y|) \equiv(|x| \leqslant|y|) \&(|x| \neq|y|),
$$

затем в класс $R \Pi_{1}$,

$$
(|x|<|y|) \equiv(\forall z)_{z \leqslant x}(|y| \neq|z|), \quad(|x| \leqslant|y|) \equiv(|x|<|y|) \vee(|x|=|y|)
$$

С использованием введенных предикатов можно доказать принадлежность классу $R \Delta_{1}$ предикатов вида

$$
\begin{aligned}
& |y|=k_{1}\left|x_{1}\right|+\ldots+k_{n}\left|x_{n}\right|+l \\
& |y|<k_{1}\left|x_{1}\right|+\ldots+k_{n}\left|x_{n}\right|+l \\
& |y|>k_{1}\left|x_{1}\right|+\ldots+k_{n}\left|x_{n}\right|+l
\end{aligned}
$$

где $k_{1}, \ldots, k_{n}$ - натуральные числа, a $l$ - целое неотрицательное число. Так, например, для класса $R \Sigma_{1}$

$$
\begin{aligned}
\left(|y|=k_{1}\left|x_{1}\right|+\ldots+k_{n}\left|x_{n}\right|+l\right) & \equiv(\exists z)_{z \leqslant y}\left(\exists v_{1}\right)_{v_{1} \leqslant x_{1}} \ldots\left(\exists v_{n}\right)_{v_{n} \leqslant x_{n}} \\
& \left(\alpha_{1}(z) \& \alpha_{1}\left(v_{1}\right) \& \ldots \& \alpha_{1}\left(v_{n}\right)\right. \\
& \&(|y|=|z|) \&\left(\left|x_{1}\right|=\left|v_{1}\right|\right) \& \ldots \ldots \&\left(\left|x_{n}\right|=\left|v_{n}\right|\right) \\
& \left.\&\left(z=v_{1}^{k_{1}} \ldots v_{n}^{k_{n}} 1^{l}\right)\right), \\
\left(|y|<k_{1}\left|x_{1}\right|+\ldots+k_{n}\left|x_{n}\right|+l\right) \equiv(\exists z)_{z \leqslant y}\left(\exists v_{1}\right)_{v_{1} \leqslant x_{1}} \ldots\left(\exists v_{n}\right)_{v_{n} \leqslant x_{n}}(\exists t)_{t \leqslant T} & \left(\alpha_{1}(z) \& \alpha_{1}\left(v_{1}\right) \& \ldots \& \alpha_{1}\left(v_{n}\right) \& \alpha_{1}(t)\right. \\
& \&(|y|=|z|) \&\left(\left|x_{1}\right|=\left|v_{1}\right|\right) \& \ldots \ldots\left(\left|x_{n}\right|=\left|v_{n}\right|\right) \\
& \left.\&\left(z t=v_{1}^{k_{1}} \ldots v_{n}^{k_{n}} 1^{l}\right)\right),
\end{aligned}
$$

где $T$ - терм $x_{1}^{k_{1}} \ldots x_{n}^{k_{n}} 1^{l}, x^{k}$ есть обозначение для конкатенации $k$ слов $x$ и $1^{l}$ при $l=0$ есть пустое слово. При переходе в класс $R \Pi_{1}$ следует учесть, что

$$
\begin{aligned}
& \neg\left(\exists z_{1}\right)_{z_{1} \leqslant T_{1}} \ldots\left(\exists z_{n}\right)_{z_{n} \leqslant T_{n}} \rho\left(x_{1}, \ldots, x_{m}, z_{1}, \ldots, z_{n}\right) \\
& \equiv\left(\forall z_{1}\right)_{z_{1} \leqslant T_{1}} \ldots\left(\forall z_{n}\right)_{z_{n} \leqslant T_{n}} \neg \rho\left(x_{1}, \ldots, x_{m}, z_{1}, \ldots, z_{n}\right)
\end{aligned}
$$

и что предикаты, входящие в правые части эквивалентностей (14) и (15), принадлежат, в частности, классу $R \Sigma_{1}$.

Пусть $B(x, y), E(x, y), P(x, y)$ - обозначения соответственно предикатов «слово $x$ является собственным началом слова $y$ », «слово $x$ является собственным концом слова $y$ », «слово $x$ входит в слово $y$ » (в последнем случае предполагается, что слово $x$ не является ни началом, ни концом слова $y$ ). Легко показать (см., например, главу 4 в [2]), что все три предиката $B, E, P$ принадлежат классу $R \Sigma_{1}$. Установим, что они входят также и в класс $R \Pi_{1}$. Например,

$$
B(x, y) \equiv(|x|<|y|) \&(\forall u)_{u \leqslant y}(\forall v)_{v \leqslant y}((u v=y) \&(|u|=|x|) \Rightarrow(u=x))
$$

Аналогичные формулы справедливы для предикатов $E(x, y)$ и $P(x, y)$. 
Для любых $n, n \geqslant 2$, и $k, 0 \leqslant k<n$, обозначим через $D_{n, k}(x)$ предикат «остаток от деления $|x|$ на $n$ равен $k »$. Справедливы соотношения

$$
\begin{aligned}
& D_{n, k}(x) \equiv(|x|=k) \vee(\exists y)_{y \leqslant x}(|x|=n|y|+k), \\
& D_{n, k}(x) \equiv(\underset{0<j<n, j \neq k}{\&}(|x| \neq j)) \&(\forall y)_{y \leqslant x} \\
& (\underset{0 \leqslant j<n, j \neq k}{\&}(|x| \neq n|y|+j)),
\end{aligned}
$$

где под $|x|=k$ понимается предикат $|x|=\left|1^{k}\right|$ (при $k=0$ этот предикат следует опустить). Таким образом, $D_{n, k} \in R \Delta_{1}$.

Приведенные выше предикаты позволяют доказать принадлежность классам $R \Sigma_{1}$ и $R \Pi_{1}$ еще целого ряда содержательно интересных предикатов. Мы не располагаем каким-либо внешним описанием классов $R \Sigma_{1}$ и $R \Pi_{1}$. Однако теорема 4 показывает, что подобное описание класса $R \Pi_{1}$ не может быть слишком простым.

В теореме 4 предполагается, что фиксирована некоторая естественная (геделевская) нумерация предикатов класса $R \Pi_{1}$. Точнее говоря, нумеруются формулы, задающие предикаты класса $R \Pi_{1}$. Имея в виду такую нумерацию, можно говорить об алгоритмических проблемах для класса $R \Pi_{1}$. В частности, будет рассмотрена проблема непустоты: по номеру предиката из класса $R \Pi_{1}$ определить, является ли данный предикат непустым (не тождественно ложным).

Теорема 4. Проблема непустоты для класса $R \Pi_{1}$ алгоритмически неразрешима.

Доказательство. Разработанная выше техника оперирования в классе $R \Pi_{1}$ дает возможность практически для любого типа абстрактных вычислительных машин эффективно по машине $\mathscr{M}$ определять в классе $R \Pi_{1}$ предикат $\operatorname{Com}_{\mathscr{M}}(x)$, который выполняется тогда и только тогда, когда $x$ представляет собой подходящим образом организованную запись вычисления на машине $\mathscr{M}$. Выбрав досточно мощный класс вычислительных устройств с неразрешимой проблемой непустоты области определения, мы получим неразрешимость проблемы непустоты для класса $R \Pi_{1}$.

Так же, как в [9], в качестве базового типа вычислительных устройств используем операторные алгоритмы специального вида. Именно, как известно (см. главу 6 в [7]), проблема непустоты области определения алгоритмически неразрешима для операторных алгоритмов, которые содержат лишь команды следующего вида: $[\times 2 ; i]$ (умножить на 2 и перейти к выполнению команды с номером $i$ ), $[\times 3 ; i]$ (аналогично предыдущей команде), [: $6 ; i, j]$ (если рассматриваемое число кратно 6 , то разделить его на 6 и перейти к выполнению команды с номером $i$, в противном случае оставить рассматриваемое число без изменения и перейти к выполнению команды с номером j), [стоп].

Пусть операторный алгоритм $\mathscr{A}$ указанного типа содержит $m$ команд, которые занумерованы числами от 1 до $m$, причем начальная команда имеет номер 1 , a единственная команда [стоп] - номер $m$. Процесс применения алгоритма $\mathscr{A}$ к числу $x$ состоит в том, что на первом шаге работы алгоритма к числу $x$ применяется команда с номером 1. При этом получается число $x_{1}$ и, кроме того, определяется номер $i_{1}$ команды, которая будет выполняться на втором шаге. Затем этот процесс повторяется: к числу $x_{1}$ применяется команда с номером $i_{1}$ и т. д. Процесс применения алгоритма $\mathscr{A}$ к числу $x$ заканчивается при выполнении команды [стоп].

Легко видеть, что при $x \neq 0$ в процессе применения алгоритма $\mathscr{A}$ к числу $x$ не может возникнуть число 0. Поэтому в целях упрощения дальнейшего изложения 
мы будем рассматривать применение операторных алгоритмов лишь к натуральным числам. Это ограничение, разумеется, не влияет на неразрешимость проблемы непустоты области определения для указанных операторных алгоритмов.

Итак, для операторного алгоритма $\mathscr{A}$ значение $\mathscr{A}(x)$ определено в том и только том случае, когда существует конечная последовательность пар чисел

$$
\left(x_{0}, i_{0}\right),\left(x_{1}, i_{1}\right), \ldots,\left(x_{t}, i_{t}\right)
$$

такая, что $x_{0}=x, i_{0}=1, i_{t}=m$, и для всякого $s, 1 \leqslant s \leqslant t, x_{s}$ есть результат применения команды с номером $i_{s-1}$ к числу $x_{s-1}$, а $i_{s}$ есть номер команды, который описанным выше способом определяется через $x_{s-1}$ и $i_{s-1}$. Последовательности (16) сопоставим слово в алфавите $\{1,2\}$

$$
1^{x_{0}} 2^{i_{o}} 1^{x_{1}} 2^{i_{1}} \ldots 1^{x_{t}} 2^{i_{t}}
$$

Эффективно по $\mathscr{A}$ построим формулу $\Phi(u)$, определяющую предикат $\operatorname{Com}_{\mathscr{A}}(u)$ из класса $R \Pi_{1}$, который выполняется только для слов вида (17). Формула $\Phi(u)$ будет представлять собой конъюнкцию формул, приводимых ниже в пп. 1-5. Для большего понимания структуры формулы $\Phi$ каждая из формул пп. 1-5 снабжена кратким комментарием.

Будем говорить, что слово $v$ является правильным началом слова $w$, если слово $v$ есть собственное начало слова $w$, то есть $w=v z$ для некоторого слова $z$, и первая буква слова $z$ отлична от последней буквы слова $v$. Аналогичным образом определяем понятия «слово $v$ является правильным концом слова $w$ и «слово $v$ правильно входит в слово $w »$.

(1) Собственным началом слова $u$ является 1 , а правильным концом - слово $2^{m}$ :

$$
B(1, u) \& E\left(12^{m}, u\right)
$$

(2) Правильным началом слова $u$ является слово вида $1^{x} 2$ (это свойство обеспечивается конъюнкцией предиката п. 1 и нижеследующего предиката):

$$
(\forall v)_{v \leqslant u}\left(\left(\alpha_{1}(v) \& B(v 2, u)\right) \Rightarrow B(v 21, u)\right)
$$

В пп. 3-5 рассматриваются только центральные вхождения слов $w 2^{i} y z$, в граничных случаях по крайней мере одна из переменных $v_{1}, v_{2}$ будет отсутствовать. Мы эти случаи отдельно не рассматриваем.

(3) Пусть $i-$ номер команды $[\times 2 ; j]$. Следующая формула утверждает, что за каждым правильным вхождением слова вида $1^{x} 2^{i}$ в слово $u$ следует правильное вхождение слова $1^{2 x} 2^{j}$ :

$$
\begin{aligned}
& \left(\forall v_{1}\right)_{v_{1} \leqslant u}\left(\forall v_{2}\right)_{v_{2} \leqslant u}(\forall w)_{w \leqslant u}(\forall y)_{y \leqslant u}(\forall z)_{z \leqslant u}\left(\left(u=v_{1} w 2^{i} y z v_{2}\right)\right. \\
& \left.\left.\quad \& \alpha_{1}(w) \& \alpha_{1}(y) \& \alpha_{2}(z) \& E\left(2, v_{1}\right) \& B\left(1, v_{2}\right)\right) \Rightarrow\left(y=w^{2}\right) \&\left(z=2^{j}\right)\right)
\end{aligned}
$$

Для каждой команды $[\times 2 ; j]$ в формулу $\Phi$ конъюнктивно входит формула этого пункта. 
(4) Пусть $i-$ номер команды $[\times 3 ; j]$. Нижеследующая формула аналогична формуле из п. 3:

$$
\begin{gathered}
\left(\forall v_{1}\right)_{v_{1} \leqslant u}\left(\forall v_{2}\right)_{v_{2} \leqslant u}(\forall w)_{w \leqslant u}(\forall y)_{y \leqslant u}(\forall z)_{z \leqslant u} \\
\left.\left(\left(u=v_{1} w 2^{i} y z v_{2}\right) \& \alpha_{1}(w) \& \alpha_{1}(y) \& \alpha_{2}(z) \& E\left(2, v_{1}\right) \& B\left(1, v_{2}\right)\right) \Rightarrow\left(y=w^{3}\right) \&\left(z=2^{j}\right)\right)
\end{gathered}
$$

(5) Пусть $i$ - номер команды $[: 6 ; j, k]$. По аналогии с пп. 3,4 формула этого пункта соответствует команде [: $6 ; j, k]$ :

$$
\begin{gathered}
\left(\forall v_{1}\right)_{v_{1} \leqslant u}\left(\forall v_{2}\right)_{v_{2} \leqslant u}(\forall w)_{w \leqslant u}(\forall y)_{y \leqslant u}(\forall z)_{z \leqslant u} \\
\left(\left(u=v_{1} w 2^{i} y z v_{2}\right) \& \alpha_{1}(w) \& \alpha_{1}(y) \& \alpha_{2}(z) \& E\left(2, v_{1}\right) \& B\left(1, v_{2}\right)\right) \\
\Rightarrow\left(D_{6,0}(w) \Rightarrow\left(w=y^{6}\right) \&\left(z=2^{j}\right)\right) \\
\left.\quad \&\left(\bigvee_{1 \leqslant l \leqslant 5} D_{6, l}(w) \Rightarrow(w=y) \&\left(z=2^{k}\right)\right)\right)
\end{gathered}
$$

Теорема доказана.

В заключение продемонстрируем некоторые возможности класса $R \Sigma_{2}$ и установим принадлежность классу $R \Sigma_{2}$ предиката $y=|x|$ логарифмического типа (ср. с леммой 4 из [4]).

Пусть $a_{n} a_{n-1} \ldots a_{1}$ - диадическое представление числа $y$. Чтобы проверить выполнение равенства $y=|x|$, достаточно образовать последовательность пар вида

$$
\left(a_{n}, 1^{a_{n}}\right),\left(a_{n} a_{n-1}, 1^{a_{n} a_{n-1}}\right), \ldots,\left(a_{n} a_{n-1} \ldots a_{1}, 1^{a_{n} a_{n-1} \ldots a_{1}}\right)
$$

и проверить, что $|x|=\left|1^{a_{n} a_{n-1} \ldots a_{1}}\right|$. Переход от пары к паре в последовательности (18) подчиняется довольно простому правилу, которое ниже формализуется средствами класса $R \Sigma_{2}$. Так же, как в теореме 4 , мы хотим перейти от последовательности (18) к слову $u$, кодирующему эту последовательность. В данном случае слово $u$ будет иметь вид

$$
2 a_{n} 21^{n} 1^{a_{n}} \bullet 2 a_{n} a_{n-1} 21^{n} 1^{a_{n} a_{n-1}} \bullet \ldots \bullet 2 a_{n} \ldots a_{2} 21^{n} 1^{a_{n} \ldots a_{2}} \bullet 2 a_{n} \ldots a_{1} 21^{n} 1^{a_{n} \ldots a_{1}}
$$

где точками - отделены подслова слова $u$, отвечающие парам последовательности (18). Нетрудно убедиться, что при условии $|x|=a_{n} \ldots a_{1}$ для длины слова $u$ имеет место оценка

$$
|u| \leqslant 2|x|+c\left(\log _{2}|x|\right)^{2}+d,
$$

где $c, d$ - некоторые константы. Следовательно, если выбрать достаточно большое $x_{0}$, то при $x>x_{0}$ будет справедливо неравенство $u \leqslant x^{3}$. Обозначим через $\lambda(u, y)$ предикат «слово $u$ имеет вид (19)». Тогда при $x>x_{0}$ справедлива эквивалентность

$$
(y=|x|) \equiv(\exists u)_{u \leqslant x^{3}}(\lambda(u, y) \& \varphi(u, x, y))
$$

где предикат $\varphi(u, x, y)$, используя слово $u$, проверяет равенство $y=|x|$. Из формулы (19) видно, что предикат $\varphi$ можно выразить следующим образом:

$$
\varphi(u, x, y) \equiv(\exists z)_{z \leqslant u}(\exists w)_{w \leqslant u}\left(\alpha_{1}(w) \&(u=z 2 w) \&(|w|=|x|+|y|)\right) .
$$


Приведенная эквивалентность показывает, что $\varphi \in R \Sigma_{1}$. Поскольку при $x \leqslant x_{0}$ значения функции $y=|x|$ можно задать табличным способом, на основании формулы (20) заключаем, что предикат $y=|x|$ будет принадлежать классу $R \Sigma_{2}$, если этому классу принадлежит предикат $\lambda$. Поэтому наши дальнейшие усилия будут направлены на доказательство включения $\lambda \in R \Sigma_{2}$.

Предикат $\lambda(u, y)$ будет определяться формулой, представляющей собой конъюнкцию формул, содержащихся в пп. 1-6. Каждый из предикатов, задаваемых этими формулами, предваряется кратким содержательным описанием. Напомним еще раз, что предикат $\lambda(u, y)$ рассматривается при достаточно больших $x$ и, следовательно, при достаточно больших $y$. В частности, можно считать, что $y>2$.

(1) Слово вида $2 a 21^{n} 1^{a} 2$, где $n=|y|$ и $a-$ первый символ диадического представления числа $y$, является собственным началом слова $u$ :

$$
\begin{aligned}
(\exists v)_{v \leqslant u}\left(\alpha_{1}(v) \&(|v|\right. & =|y|) \\
\& & (B(1, y) \Rightarrow B(212 v 12, u)) \&(B(2, y) \Rightarrow B(222 v 112, u))) .
\end{aligned}
$$

(2) Собственным концом слова $u$ является слово вида $1^{k} 2 y 21^{m}$, где $k, m>|y|$ :

$$
(\exists v)_{v \leqslant u}(\exists w)_{w \leqslant u}\left(\alpha_{1}(v) \& \alpha_{1}(w) \&(|v|>|y|) \&(|w|>|y|) \& E(v 2 y 2 w, u)\right) .
$$

(3) Если в слово $u$ входит слово $1^{n+1} 2 w 21^{n+1}$ и слово $w$ не содержит подслова $1^{n+1}$, то $|w| \leqslant|y|$ :

$$
\begin{aligned}
& (\forall w)_{w \leqslant u}(\forall z)_{x \leqslant u}\left(\left(\alpha_{1}(z) \&(|z|=|y|+1)\right.\right. \\
& \quad \& P(z 2 w 2 z, u) \&(z \neq w) \& \neg B(z, w) \& \neg P(z, w) \& \neg E(z, w)) \Rightarrow(|w| \leqslant|y|)) .
\end{aligned}
$$

(4) Если слово $1^{n+1} 2 w 21^{n+1}$ входит в слово $u$ и $|w| \leqslant|y|$, то либо $w=y$, либо $w$ есть собственное начало слова $y$ :

$$
\begin{aligned}
& (\forall w)_{w \leqslant u}(\forall z)_{z \leqslant u}\left(\left(\alpha_{1}(z) \&(|z|=|y|+1)\right.\right. \\
& \quad \& P(z 2 w 2 z, u) \&(|w| \leqslant|y|)) \Rightarrow(w=y) \vee B(w, y)) .
\end{aligned}
$$

(5) Если в слово $u$ входит слово вида $1^{n+1} 2 w_{1} 21^{m} 2 w_{2} 21^{n+1}$, где $\left|w_{1}\right| \leqslant|y|,\left|w_{2}\right| \leqslant|y|$, $m>|y|$, то $\left|w_{2}\right|=\left|w_{1}\right|+1$ :

$$
\begin{aligned}
\left(\forall w_{1}\right)_{w_{1} \leqslant u}\left(\forall w_{2}\right)_{w_{2} \leqslant u}\left(\forall z_{1}\right)_{z_{1} \leqslant u}\left(\forall z_{2}\right)_{z_{2} \leqslant u} & \\
& \left(\left(\alpha_{1}\left(z_{1}\right) \& \alpha_{1}\left(z_{2}\right) \&\left(\left|z_{1}\right|=|y|+1\right)\right.\right. \\
& \&\left(\left|z_{2}\right|>|y|\right) \&\left(\left|w_{1}\right| \leqslant|y|\right) \&\left(\left|w_{2}\right| \leqslant|y|\right) \\
& \left.\left.\& P\left(z_{1} 2 w_{1} 2 z_{2} 2 w_{2} 2 z_{1}, u\right)\right) \Rightarrow\left(\left|w_{2}\right|=\left|w_{1}\right|+1\right)\right) .
\end{aligned}
$$

(6) Если в слово $u$ входит слово вида $1^{n+1} 2 w 21^{m_{1}} 2 w a 21^{m_{2}} 2$, либо слово $u$ оканчивается на слово вида $1^{n+1} 2 w 21^{m_{1}} 2 w a 21^{m_{2}}$, где $m_{1}>|y|, m_{2}>|y|,|w|<|y|$ и $a \in\{1,2\}$, то $m_{2}=2 m_{1}+a$ :

$$
\begin{aligned}
(\forall w)_{w \leqslant u} & \left(\forall z_{1}\right)_{z_{1} \leqslant u}\left(\forall z_{2}\right)_{z_{2} \leqslant u}\left(\forall z_{3}\right)_{z_{3} \leqslant u}\left(\left(\alpha_{1}\left(z_{1}\right) \& \alpha_{1}\left(z_{2}\right) \& \alpha_{1}\left(z_{3}\right)\left(\left|z_{1}\right|=|y|+1\right)\right.\right. \\
& \&\left(\left|z_{2}\right|>|y|\right) \&\left(\left|z_{3}\right|>|y|\right) \&(|w|<|y|) \\
& \Rightarrow\left(P\left(z_{1} 2 w 2 z_{2} 2 w 12 z_{3} 2, u\right) \vee E\left(z_{1} 2 w 2 z_{2} 2 w 12 z_{3}, u\right) \Rightarrow\left(z_{3}=z_{2}^{2} 1\right)\right) \\
& \&\left(\left(P\left(z_{1} 2 w 2 z_{2} 2 w 22 z_{3} 2, u\right) \vee E\left(z_{1} 2 w 2 z_{2} 2 w 22 z_{3}, u\right) \Rightarrow\left(z_{3}=z_{2}^{2} 11\right)\right)\right) .
\end{aligned}
$$




\section{Список литературы}

1. Кузнецов А. В., К теореме о канонической форме для ординально-рекурсивных функций. В кн.: Гудстейн Р. Л., Математическая логика. ИЛ, Москва, 1961, с. 149-154.

2. Смальян Р., Теория формальных систем. Наука, Москва, 1981.

3. Непомнящий В. А., Рудиментарные предикаты и тьюринговы вычисления. Докл. $A H$ CCCP (1970) 195, №2, 282-284.

4. Непомнящий В. А., Рудиментарная интерпретация двуленточных тьюринговых вычислений. Кибернетика (1970) №2, 29-35.

5. Непомнящий В. А., Рудиментарное моделирование недетерминированных тьюринговых вычислений. Кибертетика (1973) №2, 23-29.

6. Chandra A. K., Dexter C. K., Stockmeyer L. J., Alternation. J. ACM (1981) 28, №1, 114133.

7. Мальцев А. И., Алгоритмы и рехурсивные функции. Наука, Москва, 1986.

8. Марченков С. С., О представлении словарных предикатов из арифметической иерархии. Дискретная математика (1990) 2, №1, 87-93.

9. Марченков С. С., Неразрешимость позитивной $\forall \exists$-теории свободной полугруппы. $C u$ бирский матем. журнал (1982) 23, №1, 196-198.

Статья поступила 30.03.2000. 